Pacific Journal of Mathematics

CHARACTERIZING PRIMES IN SOME NONCOMMUTATIVE 


\title{
CHARACTERIZING PRIMES IN SOME NONCOMMUTATIVE RINGS
}

\author{
HAROLD G. RUTHERFORD
}

For a ring $R$ with identity 1 , a preprime is a nonempty subset $T$ of $R$ which is closed under the two binary operations, addition and multiplication, of $R$ and with $-1 \notin T$. A prime of $R$ is a preprime of $R$ which is maximal with respect to set inclusion. A field $K$ is locally finite if every member of $K$ is a member of some finite subfield of $K$. For a finite dimensional vector space $V$ over $K$ let $\mathbb{B}=\operatorname{Hom}_{K}(V, V)$ denote the full ring of linear transformations of $V$ over $K$. Let $W$ and $L$ be subspaces of $V$ with $W \subset L \subset V$ aud $W \neq L$. Let $T(L, W)=$ $\{\alpha \in \mathbb{B} / \alpha \cdot L \subset W\}$. Then $T(L, W)$ is a preprime of $\mathbb{S}$. Let

$$
\mathscr{T}=\{T(L, W) / W, L \text { are subspaces of } V, W \subset L, W \neq L\}
$$

We will show that the primes of $\mathbb{B}$ are exactly those preprimes $T(L, W) \in \mathscr{T}$ with $\operatorname{dim}_{K} L=1+\operatorname{dim}_{K} W$.

There is also an associative monoid with zero element reminiscent of a value group for a valuation of a field. One actually finds that this monoid is independent of which prime is used to define it. However, this shows rather that while this concept yields an abelian group when the ring is commutative, it may not be the proper concept in noncommutative rings, see [1, Prop. 2.2].

A number field is a finite field extension of the field $Q$ of rational numbers. In [1, Prop. 3.4, 3.5, 3.6], Harrison has shown that for a number field $K$, the primes are exactly the useful prime divisiors of algebraic number theory and all of them when $K$ is a normal extension of $Q$. Since the definition of primes is made in arbitrary rings with 1 , it is desirous to investigate the concept for nonfields. Commutative rings have been investigated considerably in [1] and [3]. A locally finite field has no primes but $\{0\}$ (see [1, Lemma 1.4]). Thus one would expect $\mathbb{S}=\operatorname{Hom}_{K}(V, V)$ to be one of the simplest noncommutative rings to investigate.

All rings are assumed to have an identity. If $A$ and $B$ are subsets of a ring $R$ and $d$ a member of an $R$-module, $d A$ denotes $\{d a / A \in A\}, A d$ denotes $\{a d / a \in A), A \cdot B$ denotes $\{a b / a \in A, b \in B\}$ and $-A$ denotes $\{-a / a \in A\} . \quad K$ will denote a locally finite field, $V$ a finite dimensional vector space over $K$, and $\mathbb{S}=\operatorname{Hom}_{K}(V, V)$ is the ring of all $K$-linear transformations of $V$.

1. $P$-productive. A prime $P$ of a ring is called finite if $-1 \notin P$ infinite otherwise. $P$ is finite if and only if $-P \subset P$. Since the 
characteristic of $K$ is not zero, every prime of $\mathbb{S}$ is finite. Let $P$ be a finite prime of $\$$. Let

$$
B_{P}=\{\alpha \in \mathbb{S} / \alpha \cdot P \subset P\}, C_{P}=\{\beta \in \mathbb{S} / P \cdot \beta \subset P\} .
$$

We can define $A_{P}=B_{P} \cap C_{P}$ since $P$ is a finite prime. For reference see [1, Prop. 1.2, Prop. 1.3].

Definition. Let $P$ be a prime of 8 . An element $x$ of $V$ is called $P$-productive if $x \notin P x$. Here $V$ is considered as a (\$)-module by the operation $\alpha \cdot v=\alpha(u)$ for $\alpha \in \mathbb{S}, v \in V$.

Let $P$ be a prime of $\&$. We consider $K$ as embedded in $\&$ by the isomorphism which sends $k$ into $k \cdot 1_{V}$ for every $k \in K, 1_{V}$ the identity linear transformation of $V$. A straight forward generalization of Prop. 2.1 in [1] gives that if $a$ and $b$ in $K$ and their product $a b$ is in $A_{P}$, then $a \in A_{P}$ or $b \in P$. This gives the following:

Lemma 1.1. Let $P$ be a prime of S. Then $K \subset A_{P}$ and $K \cap P=\{0\}$.

Proof. For any $a \in K, a \neq 0$, there is a least positive integer $m$ with $a^{m}=1$ since $a$ is contained in a finite subfield $F$ of $K$. Now suppose $a \in K \cap P, a \neq 0$. Then $a^{m}=1, m$ a positive integer. Then $1=a^{m} \in P$ since $P$ is closed under multiplication. But since every prime of $B$ is finite, this cannot happen. So, $K \cap P=\{0\}$. Now for any $a \in K, a \neq 0, a^{m}=1$ for some least positive integer $m$. Then if $a \notin A_{P}, a^{m-1} \in P$ and also $K$ so $a^{m-1}=0$ and $a=0$ a contradiction. So $a \in A_{P}$. Thus $K \subset A_{P}$.

Proposition 1.2. Let $P$ be a prime of 85 . Then there is an element $x \in V$ which is $P$-productive.

Proof. For each $\alpha \in \mathbb{B S}$, define $V_{\alpha}=\{x \in V /(1+\alpha) \cdot x=0\} . \quad V_{\alpha}$ is easily checked to be a subspace of $V$. The dimension of $V$ over $K$ is finite so the dimension of $V_{\alpha}$ over $K$ is finite also. Among all $\alpha \in P$ choose a $\beta \in P$ with $\operatorname{dim}_{K} V_{\beta} \geqq \operatorname{dim}_{K} V_{\alpha}$ for all $\alpha \in P$. Just suppose $V_{\beta}=V$. Then $(1+\beta) x=0$ for all $x \in V$ and so $1+\beta=0$. Thus $-1=\beta \in P$ a contradiction.

Since $V_{\beta} \neq V$, there is a $c \in V$ with $c \notin V_{\beta}$. Let $d=(1+\beta) c$. Then $d$ is $P$-productive. For if not, we would have $d \in P d$. So there is a $\gamma \in P$ with $\gamma d=d$. Then for any $k \in K,(1-\gamma)(1+\beta) k c=$ $k \cdot(1-\gamma)(1+\beta) c=k(1-\gamma) d=k(d-\gamma d)=0$. Also, for any $x \in V_{\beta}$, $(1-\gamma)(1+\beta) x=(1-\gamma) 0=0$. Hence $(1+\beta-\gamma-\gamma \beta) V_{\beta}=0$ so, $V_{\beta} \subset V_{\beta-\gamma-\gamma \beta}$ and $c \in V_{\beta-\gamma-\gamma \beta}$ and $c \notin V_{\beta}$. So $\operatorname{dim}_{K} V_{\beta-\gamma-\gamma \beta}>\operatorname{dim}_{K} V_{\beta}$ which is a contradiction for $\beta-\gamma-\gamma \beta \in P$ and $\operatorname{dim}_{K} V_{\beta} \geqq \operatorname{dim}_{K} V_{\alpha}$ for all $\alpha \in P$. 
2. Some preprimes of 8 . Let $W$ and $L$ be subspaces of $V$ with $W \subset L$ and $W \neq L$. Let $T(L, W)=\{\alpha \in \mathbb{S} / \alpha L \subset W\}$. One may check that $T(L, W)$ is a preprime of $(S$. Let $\mathscr{T}=\{T(L, W) / W$ and $L$ subspaces of $V, W \subset L, W \neq L\}$. Using the fact that \&S is transitive [2, Chapter, II, §4] we can tell when two members of $\mathscr{T}$ are equal. The first step is given by

LEMMA 2.1. Let $T(L, W)$ and $T\left(L^{\prime}, W^{\prime}\right)$ be members of $\mathscr{T}$. $T\left(L^{\prime}, W^{\prime}\right) \subset T(L, W)$ if and only if $W^{\prime} \subset W \subset L \subset L^{\prime}$.

Proof. By choosing a basis of $V$ in such a way that it is the extension of a basis for proper subspaces, we will use transitivity to assert the existence of linear transformations needed to give the appropriate contradictions.

$L \subset L^{\prime}$. For if not, there is an $x \in L$ with $x \notin L^{\prime}$. Since $W \subset L$ and $W \neq L$, there is a $y \in L, y \notin W$. Thus there is an $\alpha \in$ BS with $\alpha L^{\prime}=\{0\} \subset W^{\prime}$ and $\alpha x=y \notin W$. So, $\alpha \in T\left(L^{\prime}, W^{\prime}\right)$ and $\alpha \notin T(L, W)$ a contradiction.

$W^{\prime} \subset W$. For if not, there is an $x \in W^{\prime}$ with $x \notin W$. Then since $L \subset L^{\prime}$, we may find $\beta \in$ (S) with $\beta L^{\prime}=K x \subset W^{\prime}$ and $\beta L=K x \not \subset W$. Thus $\beta \in T\left(L^{\prime}, W^{\prime}\right)$ and $\beta \notin T(L, W)$, a contradiction.

Conversely, if $W^{\prime} \subset W$ and $L \subset L^{\prime}$, one easily verifies that $T\left(L^{\prime}, W^{\prime}\right) \subset T(L, W)$.

The following corollary gives the desired criterion for equality.

CoROLLARY 2.2. Let $T\left(L^{\prime}, W^{\prime}\right)$ and $T(L, W)$ be members of $\mathscr{T}$. Then $T\left(L^{\prime}, W^{\prime}\right)=T(L, W)$ if and only if $L=L^{\prime}$ and $W=W^{\prime}$.

3. The primes in \&S. We remarked earlier that all primes of (S) are finite since the characteristic of $K$ is not 0 . Thus

THEOREM 3.1. $\mathscr{P}=\{T(L, W) / W$ and $L$ are subspaces of $V$, $\left.W \subset L, \operatorname{dim}_{K} L=1+\operatorname{dim}_{K} W\right\}$ is the set of all primes of (S).

Proof. Let $P$ be a prime of $(3)$ There is a $v \in V$ which is $P$ Productive, Prop 1.2. So $v \notin P v$. One checks that $P v$ is a subspace of $V$. Let $P v=W$ and $L=K v+W$. Now let $\alpha \in P$. Then $\alpha W \subset W$ and $\alpha v \in P v=W$. So $\alpha L \subset W$. Thus $P \subset T(L, W)$. But $T(L, W)$ is a preprime containing $P$ a maximal preprime so $P=T(L, W)$.

Conversely, let $T(L, W) \in \mathscr{P}$. Then an easy application of Zorn's lemma gives that $T(L, W)$ is contained in a prime $P$, since $T(L, W)$ is a preprime. But then there are $L^{\prime}$ and $W^{\prime}$ of $V$ with $W^{\prime} \subset L^{\prime}$ and $\operatorname{dim}_{K} L^{\prime}=1+\operatorname{dim}_{K} W^{\prime}$ and we have $T(L, W) \subset P=T\left(L^{\prime}, W^{\prime}\right)$. 
So by Lemma 2.1, $W \subset W^{\prime} \subset L^{\prime} \subset L$. But $\operatorname{dim}_{K} L=1+\operatorname{dim}_{K} W$ gives that $W=W^{\prime}$ and $L=L^{\prime}$ since $W^{\prime} \neq L^{\prime}$. Hence by Corollary 2.2, $T(L, W)=T\left(L^{\prime}, W^{\prime}\right)=P$ so $T(L, W)$ is indeed a prime.

4. The value monoid. For any prime $P$ of a ring $R$, there is a natural subring $A_{P}$ of $R$ which contains $P$. In our case $A_{P}=B_{P} \cap C_{P}$. Then one can define for each $x \in R, P: x=\{(y, z) / y, z \in R, y x z \in P\}$ and for $a$ and $b \in R$ define $a \sim b$ if $P: a=P: b$. This defines an equivalence relation on $R$. Let $[a]$ denote the equivalence class containing $a$ and let $\Gamma_{P}$ denote the set of equivalence classes of $R$. Define $\phi_{P}(\alpha)=[\alpha]$. Then $\phi_{P}: R \rightarrow \Gamma_{P}$. When $R$ is a commutative ring, $\Gamma_{P}$ is an abelian group. See [1, 11 and Prop. 2.2]. We first characterize $B_{P}$ and $C_{P}$.

LEMma 4.1. Let $P$ be a prime of (S. Then there are subspaces $W$ and $L$ of $V$ with $\operatorname{dim}_{K} L=1+\operatorname{dim}_{K} W$ and $P=T(L, W)$ by

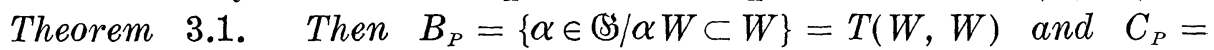
$\{\alpha \in \mathbb{S} / \alpha L \subset L\}=T(L, L)$, so that $A_{P}=T(L, L) \cap T(W, W)$.

Proof. Clearly $T(L, L) \subset C_{P}$. Now let $\alpha \in C_{P}$. Suppose $\alpha L \subset L$. Let $V=L \oplus D$. Choose $\beta \in \mathbb{S}$ with $\beta \cdot L=\{0\}, \beta(v)=v$ for all $v \in D$. Then $\beta \in P$ and $\beta \alpha L \not \subset L$, a contradiction. Hence $\alpha \in T(L, L)$ and $T(L, L)=C_{P}$. Similarly $B_{P}=T(W, W)$. Hence $A_{P}=T(L, L) \cap T(W, W)$.

Now we can be more specific that in Lemma 1.1 and give a characterization of $A_{P}$ in terms of $P$.

Proposition 4.2. Let $P$ be a prime of 8 . Then $A_{P}=P \oplus K$ as $K$-modules.

Proof. We already know that $K \subset A_{P}$ and $P \cap K=\{0\}$. Thus it only remains to show that each $\alpha \in A_{P}$ is expressible as $\beta+k, \beta \in P$, $k \in K$. Let $P=T(L, W)$ as Theorem 3.1 gives. Let $L=K v \oplus W$. Suppose $a \in A_{P}$. If $\alpha \in P, \alpha=\alpha+0,0 \in K$. So if $\alpha \notin P, \alpha L \not \subset W$ while $\alpha L \subset L$. Thus $\alpha v=k v+w, k \in K, w \in W$. Consider $\alpha-k . \quad \alpha W \subset W$ gives $(\alpha-k) W \subset W$ since $k \in A_{P}$. Also,

$$
(\alpha-k) v=\alpha v-k v=k v+w-k v=w .
$$

So $(\alpha-k) v \in W$. Thus $\alpha-k \in T(L, W)=P$. Hence

$$
\alpha=(\alpha-k)+k \in P \oplus K \text {. }
$$

Hence $A_{P}=P \oplus K$.

We are now in a position to show that the monoids corresponding to each prime $P$ of $(S)$ are all the same monoid. 
THEOREM 4.3. There is a natural associative monoid $\Gamma$ with zero element and a natural monoid homomorphism $\phi$ of $\$ 3$ onto $\Gamma$ such that for each prime $P$ of $\&, \Gamma_{P}=\Gamma$ and $\phi_{P}=\phi$.

Proof. We will outline the proof in the form of a series of claims, whose proofs are straight forward but often lengthy. We leave their proofs to the reader.

First of all we will show that if $\alpha$ and $\beta$ are in $B S$ and there is $a k \in K$ with $\beta=k \alpha$ then $\beta \sim_{P} \alpha$ where $\sim_{P}$ denotes the equivalence relation determined by any prime $P$ of (\$). For if $(\gamma, \delta) \in P: \alpha$ then $\gamma \beta \delta=\gamma k \alpha \delta=k \gamma \alpha \delta \in K P \subset P$ since $K \subset A_{P}$. So $(\gamma, \delta) \in P: \beta$. Thus $P: \alpha \subset P: \beta$. Similarly, $P: \beta \subset P: \alpha$, so $P: \alpha=P: \beta$ and $\beta \sim_{P} \alpha$.

Now if conversely we can show that if $\beta \sim_{P} \alpha$ then $\beta=k \alpha$ for some $k \in K$ we will have shown that the equivalence relations $\sim_{p}$ are really all the same and hence the monoids $\Gamma_{P}$ and monoid homomorphisms $\phi_{P}$ are all the same for each $P$. To this end, let $\beta \sim_{P} \alpha$.

Claim 1. Let $K v$ be any one-dimensional subspace of $V$. Then $\beta K v=\alpha K v$.

Proof. Let $\delta \in$ \& with $\delta L=K v, v \in V$. For $(\gamma, \delta)$ to be in $P: \alpha$, we must have $\gamma \alpha K v \subset W$. So choosing $\gamma \in \mathbb{B S}$ with this true, one gets $(\gamma, \delta) \in P: \alpha=P: \beta$ and so $\gamma \beta K v \subset W$. If $\beta K v \neq \alpha K v$, one could redefine a $\gamma_{2} \in \mathbb{S}$ with $\left(\gamma_{1}, \delta\right) \in P: \beta$ and $\left(\gamma_{1}, \delta\right) \notin P: \alpha$. This contradiction proves that $\beta K v=\alpha K v$, so long as $\beta K v \neq 0$. The similar agrument beginning with $\beta$ will then show that $\alpha K v=\beta K v$.

This gives us that if $v \in V$, there is an $a \in K$, possibly depending upon $v$, with $\beta v=a \alpha v$. We will show that $a$ is independent of the choice of $v$.

Claim 2. If $\operatorname{dim}_{K} \alpha V>1$, then for any $v_{1}$ and $v_{2}$ in $V$ which are linearly independent and $\beta v_{1}=b \alpha v_{1}$ and $\beta v_{2}=c \alpha v_{2}$, we get $b=c$.

Proof. If $\alpha v_{1}$ and $\alpha v_{2}$ are linearly independent, consider $\beta\left(v_{1}+v_{2}\right)=$ $d \alpha\left(v_{1}+v_{2}\right)$ and one finds that $b=d=c$. If not, there is a $v \in V$ with $\alpha v$ and $\alpha v_{1}$ linearly independent, since $\operatorname{dim}_{K} \alpha V>1$. Then also $\alpha v$ and $\alpha v_{2}$ are linearly independent. Consider $\beta v=e \alpha v$. Then one finds that $b=e$ and $e=c$ so again $b=c$. This proves the claim.

Claim 3. If $\operatorname{dim}_{K} \alpha V=1$, then $\operatorname{Ker} \alpha=\operatorname{Ker} \beta$ and $\alpha V=K v$ for some $v \in V$ and $\beta V=K v$ also. In addition, if $v^{\prime} \in V, v^{\prime} \notin \operatorname{Ker} \alpha$, then $\beta^{\prime} v=b \alpha v^{\prime}$ and indeed $\beta=b \alpha$.

Proof. For $x \in \operatorname{Ker} \alpha, \beta x=c \alpha x$ for some $c \in K$ and $\alpha x=0$ so 
$\beta x=0$ so $\operatorname{Ker} \alpha \subset \operatorname{Ker} \beta$. By dimension arguments, $\operatorname{Ker} \alpha=\operatorname{Ker} \beta$. $\beta V=\alpha V$ by the Claim 1. Now any $w \in V$ is $w=x+k v^{\prime}, x \in \operatorname{Ker} \alpha=$ $\operatorname{Ker} \beta$ and $k \in K$. Then one checks that $\beta w=k \beta v^{\prime}=b k \alpha v^{\prime}=b \alpha w$ since $a \alpha x=0=\alpha x$. Thus $\beta=b \alpha$.

Combining the last two claims, we see that if $\operatorname{dim}_{K} \alpha V=1, \beta=\alpha \alpha$ for some $a \in K$. If $\operatorname{dim}_{K} \alpha V>1$, then we showed $a$ is independent of the choice of $v \in V$ used to define it. Hence again $\beta=a \alpha$. This concludes the proof that if $\beta \sim_{P} \alpha$, then $\beta=a \alpha, a \in K$.

Sincere thanks are due David Harrison for his constant inspiration while this work was being done. Few are able to transmit their enthusiasm to students as well as he, at least it is difficult to conceive of someone doing it better. I would also like to thank Merle Manis for many informative conversations as well as his computations in matrices which served as guidelines for my characterizations. Thanks are also due to the Health, Education, and Welfare Department who provided support for three years as an N.D.E.A. Fellow at New Mexico State University and to the National Science Foundations who provided support one year as an N.S.F. Trainee at the University of Oregon. Without their aid, individuals who produce well at home would be hard pressed to produce well mathematically.

\section{BIBLIOGRAPHY}

1. D. K. Harrison, Finite and infinite primes for rings and fields, Amer. Math. Soc. Memoirs, No. 68, 1967.

2. N. Jacobson, Structures of Rings, Amer. Math. Soc. 1956.

3. M. Manis, Valuations on a commutative rings, $\mathrm{Ph}$. D. thesis, University of Oregon, 1966.

Received September 21, 1967. 


\section{PACIFIC JOURNAL OF MATHEMATICS}

\section{EDITORS}

\section{H. ROYDEN}

Stanford University

Stanford, California

\author{
R. R. Phelps \\ University of Washington \\ Seattle, Washington 98105
}

\section{J. DugundJI}

Department of Mathematics University of Southern California Los Angeles, California 90007

\section{RICHARD ARENS}

University of California

Los Angeles, California 90024

\section{ASSOCIATE EDITORS}

\section{E. F. BECKENBACH}

B. H. NEUMANN

F. WOLF

K. YOSIDA

\section{SUPPORTING INSTITUTIONS}

UNIVERSITY OF BRITISH COLUMBIA CALIFORNIA INSTITUTE OF TECHNOLOGY UNIVERSITY OF CALIFORNIA MONTANA STATE UNIVERSITY UNIVERSITY OF NEVADA NEW MEXICO STATE UNIVERSITY OREGON STATE UNIVERSITY UNIVERSITY OF OREGON OSAKA UNIVERSITY UNIVERSITY OF SOUTHERN CALIFORNIA

\author{
STANFORD UNIVERSITY \\ UNIVERSITY OF TOKYO \\ UNIVERSITY OF UTAH \\ WASHINGTON STATE UNIVERSITY \\ UNIVERSITY OF WASHINGTON \\ AMERICAN MATHEMATICAL SOCIETY \\ CHEVRON RESEARCH CORPORATION \\ TRW SYSTEMS
}

NAVAL WEAPONS CENTER

Mathematical papers intended for publication in the Pacific Journal of Mathematics should be in typed form or offset-reproduced, double spaced with large margins. Underline Greek letters in red, German in green, and script in blue. The first paragraph or two must be capable of being used separately as a synopsis of the entire paper. It should not contain references to the bibliography. Manuscripts, in duplicate if possible, may be sent to any one of the four editors. All other communications to the editors should be addressed to the managing editor, Richard Arens, University of California, Los Angeles, California 90024.

Each author of each article receives 50 reprints free of charge; additional copies may be obtained at cost in multiples of 50 .

The Pacific Journal of Mathematics is published monthly. Effective with Volume 16 the price per volume (3 numbers) is $\$ 8.00$; single issues, $\$ 3.00$. Special price for current issues to individual faculty members of supporting institutions and to individual members of the American Mathematical Society: $\$ 4.00$ per volume; single issues $\$ 1.50$. Back numbers are available.

Subscriptions, orders for back numbers, and changes of address should be sent to Pacific Journal of Mathematics, 103 Highland Boulevard, Berkeley 8, California.

Printed at Kokusai Bunken Insatsusha (International Academic Printing Co., Ltd.), 7-17, Fujimi 2-chome, Chiyoda-ku, Tokyo, Japan.

PUBLISHED BY PACIFIC JOURNAL OF MATHEMATICS, A NON-PROFIT CORPORATION

The Supporting Institutions listed above contribute to the cost of publication of this Journal, but they are not owners of publishers and have no responsibility for its content or policies. 


\section{Pacific Journal of Mathematics}

\section{Vol. 27, No. $2 \quad$ February, 1968}

Leonard E. Baum and George Roger Sell, Growth transformations for

functions on manifolds ............................ 211

Henry Gilbert Bray, A note on CLT groups ................... 229

Paul Robert Chernoff, Richard Anthony Rasala and William Charles

Waterhouse, The Stone-Weierstrass theorem for valuable fields....... 233

Douglas Napier Clark, On matrices associated with generalized

interpolation problems ................................

Richard Brian Darst and Euline Irwin Green, On a Radon-Nikodym theorem for finitely additive set functions . ...................... 255

Carl Louis DeVito, A note on Eberlein's theorem..................... 261

P. H. Doyle, III and John Gilbert Hocking, Proving that wild cells exist . . . 265

Leslie C. Glaser, Uncountably many almost polyhedral wild $(k-2)$-cells in

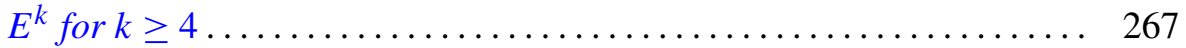

Samuel Irving Goldberg, Totally geodesic hypersurfaces of Kaehler

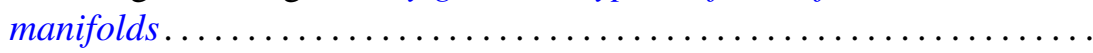

Donald Goldsmith, On the multiplicative properties of arithmetic functions .................................... 283

Jack D. Gray, Local analytic extensions of the resolvent ............ 305

Eugene Carlyle Johnsen, David Lewis Outcalt and Adil Mohamed Yaqub,

Commutativity theorems for nonassociative rings with a finite division ring homomorphic image ....................

André (Piotrowsky) De Korvin, Normal expectations in von Neumann

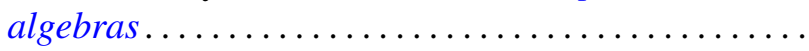

James Donald Kuelbs, A linear transformation theorem for analytic

Feynman integrals..........................

W. Kuich, Quasi-block-stochastic matrices ................... 353

Richard G. Levin, On commutative, nonpotent archimedean

semigroups ............................... 365

James R. McLaughlin, Functions represented by Rademacher series ... . . . 373

Calvin R. Putnam, Singular integrals and positive kernels............ 379

Harold G. Rutherford, II, Characterizing primes in some noncommutative

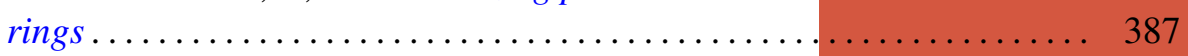

Benjamin L. Schwartz, On interchange graphs................... 393

Satish Shirali, On the Jordan structure of complex Banach *algebras . . . . . 397

Earl J. Taft, A counter-example to a fixed point conjecture............. 405

J. Roger Teller, On abelian pseudo lattice ordered groups ..... 